\title{
FSP AND FLTL FRAMEWORK FOR SPECIFICATION AND VERIFICATION OF MIDDLE-AGENTS
}

\author{
AmELIA BĂDIC $\breve{A} *$, Costin BĂDIC $\breve{A} * *$ \\ ${ }^{*}$ Business Information Systems Department \\ University of Craiova, 13 A.I. Cuza St, 200530, Craiova, Romania \\ e-mail:ameliabd@yahoo.com \\ ${ }^{* *}$ Software Engineering Department \\ University of Craiova, 107 Decebal Blvd, 200440, Craiova, Romania \\ e-mail: costin.badica@software.ucv.ro
}

\begin{abstract}
Agents are a useful abstraction frequently employed as a basic building block in modeling service, information and resource sharing in global environments. The connecting of requester with provider agents requires the use of specialized agents known as middle-agents. In this paper, we propose a formal framework intended to precisely characterize types of middle-agents with a special focus on matchmakers, brokers and front-agents by formally modeling their interactions with requesters and providers. Our approach is based on capturing interaction protocols between requesters, providers and middle-agents as finite state processes represented using FSP process algebra. The resulting specifications are formally verifiable using FLTL temporal logic. The main results of this work include (i) precise specification of interaction protocols depending on the type of middle-agent (this can also be a basis for characterizing types of middle-agents), (ii) improvement of communication between designers and developers and facilitation of formal verification of agent systems, (iii) guided design and implementation of agent-based software systems that incorporate middle-agents.
\end{abstract}

Keywords: multi-agent system, interaction protocol, process algebra, formal specification.

\section{Introduction}

Sharing information, resources and services on a global scale is a key function of the modern information society. Advanced digital technologies including software agents, grids and Web services have recently been proposed to support this function. In this context, the ability of parties involved (humans, businesses or software) to flexibly discover each other and to dynamically engage in relationships is very important.

Agents are a useful abstraction frequently employed as a basic building block in modeling service, information and resource sharing in global environments. Typically such an environment contains requester and provider agents that are dynamically created and destroyed and consequently do not know each other in advance. Connecting requester agents with provider agents is a crucial issue known as the connection problem (Decker et al., 1997). Its solution requires the use of additional specialized agents known as middle-agents (Decker et al., 1997; Yarom et al., 2003; Klusch and Sycara, 2001).
The activity carried out by middle-agents is called intermediation, and it assumes suitable interactions of middleagents with requesters and providers.

The main contribution of this paper is precise characterization of types of middle-agents-matchmakers, brokers and front-agents-by formally modeling their interactions with requesters and providers in global environments. The paper employs a formal framework for the modeling and analysis of interaction protocols between requesters, providers and middle-agents. In this framework we focus on interactions, i.e., sequences of messages exchanged between parties involved-requesters, providers and middle-agents. In our opinion the results of this work have the following benefits: (i) precise specification of the interactions depending on the type of middle-agent (this can also be a basis for characterizing types of middleagents); (ii) understanding the requirements of parties involved in such interactions; (iii) improvement of communication between designers and developers and facilitation of formal verification; (iv) guided design and implemen- 
tation of software systems that incorporate middle-agents.

Despite the early work of Decker et al. (1997), which advocates systematic classification of middle-agents, we noticed that "notions of middle-agents, matchmakers, brokers $[\ldots]$ are used freely in the literature [...] without necessarily being clearly defined" (Klusch and Sycara, 2001). Moreover, most of the references to middleagents focus in more or less detail on various applications and systems that use different types of middle-agents, highlighting implementation and/or performance issues. Therefore, in this paper, we focus on improving this stateof-affair by presenting and discussing formal models of middle-agents with the goal of highlighting their similarities and differences.

Typical use of middle-agents is encountered in ecommerce applications (Bădică et al., 2007; Fasli, 2007; Yarom et al., 2003). For example, the model agent-based e-commerce system discussed by Bădică et al. (2007) uses middle-agents to connect user buyers on the purchasing side with shops on the selling side in a distributed marketplace. Each user buyer is represented by a Client agent, and each shop is represented by a Shop agent. The user buyer submits an order to the system for purchasing a product via his or her Client agent. The Client agent acts as a Front-agent (see Section 5) with respect to connecting the user buyer with an appropriate Shop agent that provides the requested product. Moreover, the Client agent uses a special agent called the Client Information Center (CIC) that is responsible for providing information which shop in the system sells which products. Thus, it can be easily noticed that the CIC is in fact a Matchmaker (see Section 5) with respect to connecting the Client agent with an appropriate Shop agent.

Matchmakers are also available in general-purpose agent platforms. For example, the Directory Facilitator $(D F)$ is an optional component of an FIPA-compliant agent platform 1 that is present in agent platforms like JA$\mathrm{DE}$. The $D F$ provides a yellow pages service allowing agents to publicize, unregister and update descriptions of services that are made available for use to other agents in the system.

Formalizing software architectures, components, agents and services has generated a lot of interest during the last years (Zhang et al., 2010). Many approaches utilize process algebras as the foundational formalism (Bergstra et al., 2001). We follow the trend by grounding our framework for the modeling and analysis of interactions between requesters, providers and middle-agent on finite state process (FSP) algebra (Magee and Kramer, 2006). The approach is applied to middle-agent types proposed by Decker et al. (1997). Then we show how qualitative properties of their models can be formal-

${ }^{1}$ http://www.fipa.org.

${ }^{2}$ http: //jade.cselt.it. ly defined and verified using fluent linear temporal logic (FLTL) (Magee and Kramer, 2006). Our work can be described as an FSP and FLTL-based framework for formal specification and verification of systems with middleagents. Note that, concerning the specification of software systems, to the best of our knowledge FSP has only been used for the specification of software architectures, processes, components, services, and aspects (Bădică et al., 2003; Foster et al., 2006; Hennicker and Ludwig, 2005; Xu et al., 2009), and it is only recently that FSP has been applied to model software agent interactions (Bădică and Bădică, 2008c).

Our proposal for using FSP and FLTL for this task is motivated by the following: (i) FSP and FLTL are formal specification and verification languages that have a rigorous mathematical foundation; (ii) FSP has a clear and concise syntax and compositional semantics, thus being very appropriate to concisely describe the behavior and interactions in systems with middle-agents; (iii) the definition of properties in FLTL can benefit from earlier work in verification patterns (Dwyer et al., 1999); (iv) FSP and FLTL are also well supported by the Labeled Transition System Analyzer (LTSA) software tool (Magee and Kramer, 2006); (v) FSP has operational semantics, so it follows that the resulting specifications are executable (can be traced with the help of LTSA); moreover, FSP specifications can be used as a basis for design and software implementation of agent behaviors, for example using finite state machines (Goh et al., 2007); (vi) recently, FSP has been extended to Modal Transition Systems formalism (MSP) for modeling and analysis in the presence of partial information about system behavior; MSP is currently supported by the Modal Transition System Analyzer tool (MTSA); MSP and MTSA provide better support in the context of current software development practices (D'Ippolito et al., 2008).

We start in Section 2 with a literature overview of related works on middle-agents and process-algebraic approaches to the formalization of agent systems. In Section 3 we state the basic assumptions underlying our modeling. In Section 4 we introduce FSP and the guidelines of modeling agent interactions with FSP. Then, in Section 5, we present detailed FSP models of Matchmaker, Front-agent, and Broker middle-agents. In Section 6 we show how FLTL can be used to formally check the developed models against qualitative properties. We follow in Section 7 with experimental evaluation and verification of the models. The final part of the paper contains our conclusions.

\section{Related works}

Research literature referring to middle-agents is quite rich: a search on Google for the words "middle-agents" returns about 20000 references. Despite this relatively large num- 
ber of references, which indicates that there is a growing interest in the subject of middle-agents, our literature overview indicates that only very few papers address the problem of a concise definition of middle-agents in terms of their interaction capabilities with provider and requester agents, to precisely characterize their typology. Rather, many references to middle-agents focus in more or less detail on various applications and systems that use different types of middle-agents, most often brokers and/or matchmakers (see Klusch and Sycara, 2001), and highlighting implementation and/or performance issues.

The starting point of our work is the classification of middle-agents introduced in the seminal work of Decker et al. (1997). Based on privacy assumptions about what is initially known in an interaction by requesters, middleagents, and providers about requester preferences and provider capabilities, Decker et al. (1997) proposed nine types of middle-agents: Broadcaster, Matchmaker, Frontagent, Anonymizer, Broker, Recommender, Blackboard, Introducer, and Arbitrator (see Table 1). Note that in this context the term "initially" means "before the interaction between the requester and the provider".

Wong and Sycara (2000) attempt to propose a more systematic classification of middle-agents by refining the classification introduced by Decker et al. (1997). The authors suggested the use of six binary dimensions for characterizing middle-agent types. However, while noting that not all $2^{6}=64$ combinations are meaningful, Wong and Sycara (2000) do not clearly show how to appreciate which are meaningful and which are not, leaving this decision up to the designer's intuition. After reviewing that taxonomy, our conclusion is that, on the one side not all proposed dimensions are relevant for our work, and, on the other, that we do not totally agree with that taxonomy. For example, according to Dimension 1, Wong and Sycara (2000) claim that "providers and requesters should act in a complementary way with respect to who sends information to middle-agents, i.e., if providers push, requesters pull and vice-versa". In our opinion the main characteristic of a Broker is that both providers and requesters push, while the Broker has the option to choose the most effective matching provider capability for a given request and the most effective matching requester preference for a given provider capability. Obviously, the taxonomy proposed by Wong and Sycara (2000) fails to correctly describe such a behavior.

Alagar and Holliday (2002) suggest the use of labeled transition systems (LTSs) for modeling agent types. However, this paper does not address the formal modeling of middle-agents. Klusch and Sycara (2001) discuss the modeling of matchmakers and brokers using input/output automata (following the initial proposal of Wong and Sycara (2000)), while Hristozova and Sterling (2003) informally describe an application of middle-agents and ontologies in the area of value-added publishing.
Our "agents-as-processes" modeling approach is not entirely new. Esterline et al. (2006) propose the use of $\pi$ calculus (Milner, 1999) and show models of a prototype agent system called LOGOS for an unattended grounds operation-center using a fault resolution scenario. However, while the authors show how to use a software tool for checking the proposed specifications, they do not provide any experimental results — only general modeling guidelines are given. Rouff et al. (2006) also focus on the LOGOS agent system, but using CSP process algebra (Hoare, 1985). While that paper discusses some benefits of the method (detecting race conditions, message omissions, and better understanding of the system), it still does not provide any experimental results and does not discuss the practical problems encountered.

Bădică et al. (2003) propose a formal model of business processes captured as role activity diagrams, using FSP. Another work by Bădică et al. (2007) introduces a general framework for FSP modeling of middle-agents and shows how this framework can be used to model a $R e$ commender agent. Using the same idea, Bădică and Bădică (2008c) present a model of an Arbitrator middle-agent for coordination of participants in single-item English auctions. Another work reported by Bădică and Bădică (2008b) is an extension of the research by Bădică et al. (2007) as well as Bădică and Bădică (2008c) for modeling Matchmaker, Front-agent and Broker middle-agents, including both theoretical and experimental results. The papers by Bădică and Bădică (2008a; 2008e; 2009a) focus on introducing verification models of systems that contain middle-agents, using FLTL temporal logic. Bădică and Bădică (2008d) show how models of Matchmaker (Bădică and Bădică, 2008b) and Arbitrator for English auctions (Bădică and Bădică, 2008c) can be combined into a more complex system for agent-based auctions with matchmaking capabilities. Moreover, in another work (Bădică and Bădică, 2009b), we extend the auction model of Bădică and Bădică (2008c) to formally specify and verify the agent-based auction service discussed by Dobriceanu et al. (2009) using FSP and FLTL. Finally, in a recent paper (Bădică et al., 2009), we show how our formal framework can be applied to model protocol-based service coordination in agent systems. Actually, this coordination approach is a natural extension of the service model introduced by Bădică and Bădică (2009b).

A limitation of our process-algebraic method that we would like to address in the future is that it does not provide features for modeling intelligence aspects of middleagents, i.e., their decision making, strategic and reasoning capabilities. In our opinion this would require the extension of the models with abilities for representing state variables, utilities and knowledge. Some related works in this area include those by Merayo et al. (2007) as well as Miller and McBurney (2007). Merayo et al. (2007) introduce an extension of finite state machines-extended 
Table 1. Middle-agent types.

\begin{tabular}{|c|c|c|c|}
\hline Preferences/Capabilities & Provider only & Provider \& MA & All \\
\hline \hline Requester only & Broadcaster & Front-agent & Matchmaker \\
\hline Requester \& MA & Anonymizer & Broker & Recommender \\
\hline All & Blackboard & Introducer & Arbitrator \\
\hline
\end{tabular}

utility state machines (EUSMs) for modeling strategic aspects of intelligent agents. The EUSM supports a richer representation of agents behavior as compared to the FSP approach by enhancing their state representation with state variables and utility functions. Miller and McBurney (2007) propose a process algebraic approach enhanced with constraint-reasoning capabilities- $\mathcal{R} \mathcal{A S} \mathcal{A}$ for the specification of semantic aspects of agent interaction protocols. Using $\mathcal{R} \mathcal{A} \mathcal{S} \mathcal{A}$, intelligent agents can reason at run-time about their possible actions and effects in order to tune their performances. However, Miller and McBurney (2007) only present a simplistic example and it is not clear when and how the $\mathcal{R} \mathcal{A} \mathcal{S} \mathcal{A}$ framework should be applied in practice. Finally, Rahimi et al. (2002) propose an extension of higher-order $\pi$-calculus to represent the intelligence component of an autonomous agent.

As for as their application areas are concerned, middle-agents were recently put forward by Fasli (2007) in the context of e-commerce. The author covers in some detail Matchmaker, Broker, Broadcaster and Recommender with a focus on interaction protocols and languages for describing requester preferences and provider capabilities. However, while the Matchmaker description fits within the models that we propose in our paper, note that Broker described by Fasli (2007) actually corresponds to our model of Front-agent.

Communities of middle-agents were also used for self-organization of distributed information systems according to dynamically changing user preferences and characteristics (Wang, 2002). The focus of this work was set on flexibility and scalability issues, rather than formal modeling and understanding of middle-agent typology.

\section{Modeling assumptions}

In this section we introduce the basic modeling assumptions that we employ in our modeling and analysis of interactions between requesters, providers and middle-agents. These assumptions include (i) abstracting away from domain and language specific details, and (ii) taking into account the distinction between subscriptions and ordinary requests.

3.1. Domain and language specific details. There is certainly a domain dependent component of intermediation, involving domain or language specific aspects of pre- ferences and capabilities of requesters and providers

For example, there are clearly domain-specific differences between searching and intermediation of business or holidays travel packages in an e-travel environment and discovering learning resources (for example, a programming course) for an e-learning application. However, we would expect that interactions necessary to search for a travel package that has a given set of characteristics by querying travel agencies and to search for a programming course whose completion would guarantee a certain level of competence in a given programming language are basically the same.

Following this idea, in our framework we abstract away from (i) the specific and domain dependent details of the environment, i.e., we do not care if the target of our analysis is, for example, related to learning, commerce or travel, and (ii) actual languages used for representing requests, responses, preferences, and capabilities, i.e., we do not care if requests and responses are for example, represented using FIPA ACL 3 and capabilities and preferences using FIPA SL4. In other words, we abstract away from the matching activity and, instead, we focus on the dynamics of interactions, i.e., sequences of messages exchanged between involved parties-requesters, providers and middle-agents.

One apparent weakness introduced by adopting this assumption is setting the focus on control modeling details (agents and their interactions), rather than on data modeling details. This claim is supported by the observation that the goal of real world implementations of agent systems with middle-agents is usually mediation and data transfer. While abstraction from data type specification can be of course limiting for the achievement of the goal of precise specification and design of an agent system as part of a software development process, this abstraction can be extremely useful for characterizing types of middle-agents and understanding the interaction requirements of parties involved. Moreover, if we also consider the goal of formal verification of agent interactions, data abstraction can be extremely useful for reducing the statespace explosion in model checking (Zhang et al., 2010). Finally, note that there are proposals to enhance process algebraic approaches with the ability of handling data by means of equational abstract data types, like $\mu C R L$, de-

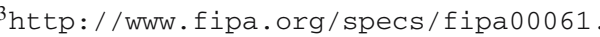
http://www.fipa.org/specs/fipa00008. 
scribed in detail by Fokkink (2007). However, this approach is outside the scope of this paper and therefore we will not pursue it here.

3.2. Subscriptions versus ordinary requests. Following a recent work of Mbala et al. (2006), we found it useful to distinguish from the very beginning between subscriptions and ordinary requests. Subscriptions can be defined as long-lived or reproducible "requests" (Mbala et al., 2006), while ordinary requests are non-reproducible (simply called requests in what follows). The difference is that a successful subscription will be continually served until it is eventually canceled (by the requestersubscriber in this particular case), while an ordinary request is issued and served at most once or eventually abandoned (by the middle-agent). For example, a buyer agent can submit a request to a directory agent that manages information about seller agents and the categories of products they sell to find out a list of potential sellers of a given product. Once issued, the request is served by providing the list of sellers and the conversation between the buyer agent and the directory agent ends. Differently, a travel agent can subscribe to a weather information provider that periodically sends weather information to all her subscribers.

Note that the distinction between requests and subscriptions is related to serving requests either in pull or in push mode (Wong and Sycara, 2000). A request served in pull mode is either immediately served or declined by the middle-agent, while a request served in push mode is memorized and the requester notified later if the request can be served or it is abandoned by the middle-agent.

The situation is symmetric from the point of view of providers. In this case, a provision (of services, resources or information) can be either non-reproducible or reproducible. In the first case, the provision, once consumed, is no longer available until explicitly made so by the provider, while in the second case the provision is continuously available until explicitly canceled by the provider. An example of a non-reproducible provision is a seller that sells a book on eBay-once the book is sold, it will no longer be available for sale. An example of a reproducible provision is a weather information provider like Weather Underground.

Note that reproducibility and non-reproducibility of requests and provisions will affect the interaction patterns between requesters, providers and middle-agents. As the focus of our work is to precisely characterize middleagents types as proposed by Decker et al. (1997), we had to assume that requests are non-reproducible (i.e., we focus on requests, and not subscriptions) and that provisions are reproducible. However, the other situations are also conceivable, leading obviously to different models.

\section{Modeling framework}

Before presenting FSP models of middle-agents we first briefly review FSP and then we introduce the guidelines of our modeling approach.

4.1. Overview of FSP. FSP is an algebraic specification technique of concurrent and cooperating computational processes as finite state labeled transition systems (LTSs hereafter). FSP allows a more compact and easy to manage description of an LTS, rather than by directly describing it as a list of states and transitions between states.

An FSP model consists of a finite set of sequential and/or composite process definitions. Additionally, a sequential process definition consists of a sequence of one or more definitions of local processes. A process definition consists of a process name associated with a process term.

FSP uses a rich set of constructs for process terms (see Magee and Kramer, 2006) . For the purpose of this paper we are using the following constructs: (i) action prefix $(a \rightarrow P)$, nondeterministic choice $(P \mid Q)$, and process alphabet extension $\left(P+\left\{a_{1}, \ldots, a_{n}\right\}\right)$ for sequential process terms, and (ii) parallel composition $(P \| Q)$ and relabeling $\left(P /\left\{\right.\right.$ new $_{1} /$ old $_{1}, \ldots$, new $_{k} /$ old $\left.\left._{k}\right\}\right)$ for composite process terms.

FSP has operational semantics given via an LTS. The mapping of an FSP term to an LTS is described in detail by Magee and Kramer (2006), and it follows the intuitive meaning of FSP constructs.

4.2. Modeling guidelines. We propose the following guidelines to be applied in the development of FSP models of middle-agents:

(i) Agents are modeled as FSP processes. As our process language is FSP, we chose to model agents types as sequential processes. Instantiation of an agent of a given type can be defined by invoking the associated process with a suitable relabeling of its alphabet.

(ii) A multi-agent system is modeled as a parallel composition of processes. It follows that communication between agents is modeled by appropriately utilizing the synchronization capabilities of FSP. In FSP, synchronization of processes of a parallel composition is done by default on their common alphabets. Accordingly, special care should be taken in order to accurately model agent communication using FSP synchronization. Depending on circumstances this will require relabeling and/or alphabet extension of local processes (see, for example, the Matchmaker model from Fig. 1). Relabeling is utilized when a new process is instantiated via its process name, so basically it is a reusability mechanism. Alphabet extension is utilized to correctly model parameter passing between processes via action synchronization. This modeling is not so obvious, 
and therefore we explain it by means of an example. Let us suppose that a process $Q$ must pass a parameter $v$ with the value in a finite set $\mathcal{V}$ to a process $P$. The decision of what value is passed by $Q$ to $P$ is taken by $Q$ and usually only some of the values (not all) in $\mathcal{V}$ can be passedlet us denote this set of values by $\mathcal{V}^{\prime} \subseteq \mathcal{V}$. This situation is modeled by a set of actions $\operatorname{send}(v)$ indexed with all the values of $v$ that can be set by $Q$ (i.e., values from the set $\left.\mathcal{V}^{\prime}\right)$. Actions $\operatorname{send}(v)$ must be performed by both $P$ and $Q$, but because $P$ does not know the value of $v$ that is passed by $Q$, it must be able to execute all the actions $\operatorname{send}(v \in \mathcal{V})$, so all of them will be automatically included in its alphabet. As $Q$ will execute an action $\operatorname{send}(v)$ such that $v$ is a value that must be passed to $P$, it follows that only actions $\operatorname{send}\left(v \in \mathcal{V}^{\prime}\right)$ will be part of $Q$ 's alphabet. Communication between $P$ and $Q$ is modeled by considering the parallel composition of $P$ and $Q$. Note that if $v \in \mathcal{V}^{\prime}$ then action synchronization will correctly model passing of $v$ from $Q$ to $P$. However, if $v \in \mathcal{V} \backslash \mathcal{V}^{\prime}$, the process $P$ will be able to execute $\operatorname{send}(v)$, while $Q$ will not, so execution of $P \| Q$ will proceed without synchronization with action $\operatorname{send}(v)$. This incorrect behavior can be corrected by extending the alphabet of $Q$, so instead of $Q$ we shall use $Q^{\prime}=Q+\{\operatorname{send}(v \in \mathcal{V})\}$. In this situation, if $v \in \mathcal{V} \backslash \mathcal{V}^{\prime}$, then the processes $P$ and $Q^{\prime}$ must synchronize on their common alphabets, and independent execution of $P$ without synchronization with $Q^{\prime}$ will not be possible for actions $\operatorname{send}(v)$ where $v \in \mathcal{V} \backslash \mathcal{V}^{\prime}$.

Note that alphabet extension is utilized in the Matchmaker model (see Section 5.1).

(iii) We assume that $\mathcal{R}$ is the set of requesters and $\mathcal{P}$ is the set of providers. The identity of requester and provider agents is explicitly represented by integer indexes $r \in \mathcal{R}$ and $p \in \mathcal{P}$.

(iv) Requests made by requester agents are indexed with the requester ID. Requests made to the providers are indexed with the provider ID. It follows that (a) requests from requesters to middle-agents are indexed only with the ID of the requester-action request $(r)$, and (b) requests from middle-agents to providers are indexed only with the ID of the providers; (c) requests made by requesters directly to providers are indexed with both the IDs of the requester and the provider-action request_to_provider $(r, p)$. Note that details like request preferences and service capabilities are abstracted away from our models, as we consider them unnecessary to understand the specific particularities of interaction for each type of middle-agent. Accordingly, this assumption means that details of a request/capability are "incorporated" in the ID of the requester/provider.

(v) The matching operation is modeled as a relation $\mathcal{M}$ between the sets $\mathcal{P}$ of providers and $\mathcal{R}$ of requesters, i.e., $\mathcal{M} \subseteq \mathcal{P} \times \mathcal{R}$. If $r$ is a requester ID then the set $P$ of IDs of matching providers that results from a matching operation is $P=\mathcal{M}(r)$. Symmetrically, if $p$ is a provider ID then the set $R$ of the IDs of matching requesters that results from a matching operation is $R=\mathcal{M}^{-1}(p)$.

(vi) We define some action naming conventions with the role of standardizing the communication interfaces of the agents with the exterior environment consisting of requesters, providers, and possibly other middle-agents. A provider will use the action offer to register a capability and action withdraw to unregister a capability with the middle-agent. As for the provider's service interface, we use the convention receive_request / send_reply to model the receipt of a service request and return of a reply. A requester will use the action request for requesting a service from the middle-agent. If the service is not intermediated by the middle-agent, then the middle-agent will respond with the action tell. If the service is intermediated by the middle-agent, then the middle-agent will respond either with the action success if the serve provision was successful or with the action fail in the case of failure to service the requester.

\section{FSP models of Matchmaker, Front-agent and Broker}

Based on our literature overview, we noticed that frequently utilized middle-agents for connecting provider and requester agents are Matchmaker, Front-agent, and Broker. In this section, we present and discuss their FSP models, using the framework and the modeling assumptions introduced in the previous sections. Taking into account that the modeling principles are general and the resulting models are complex enough, one can easily apply this modeling approach to the other types of middle-agents (see, e.g., the model of Recommender discussed by Bădică et al . (2007)).

Note that we start with an intuitive description of types of middle-agents that aims at providing a concise natural language description of middle-agent interaction patterns with requesters and providers, emphasizing the initial assumptions that were used for their classification (Decker et al., 1997) and follow with the specification and analysis of their formal FSP models.

5.1. Matchmaker middle-agent in FSP. A Matchmaker middle-agent (also known as Yellow-pages, see Table 11) assumes that, before the interaction between the requester and the provider, requester preferences are known only to the requester, while provider capabilities are or will become known to all interaction participants. This means that a provider will have to advertise its capabilities with Matchmaker, and Matchmaker is responsible for matching a request with registered capabilities advertisements. However, the fact that provider capabilities are initially known also by the requester means that the result of the matching (i.e., the set of matching providers) will be 
returned by Matchmaker to the requester (provider capabilities become thus known to the requester), and the choice of the matching provider is the responsibility of the requester. Consequently, the transaction is not intermediated by Matchmaker, as would be the case, for example, with Broker or Front-agent.

The block diagram and FSP specification of a system with requesters, providers and Matchmaker are shown in Fig. 1.

The Provider agent registers its capability offer (the action offer) with Matchmaker and then enters a loop where it receives requests from Requester agents via the action receive_request and processes and replies accordingly via the action send_reply. Note that Provider can also withdraw a registered capability offer, and while its capability is not registered it always refuses to serve a request (the action refuse_request).

The Requester agent submits a request to Matchmaker (the action send_request) and then waits for a reply. Matchmaker replies with a set of matching providers (the action tell with the argument $\mathcal{M}(r) \cap P$ representing the set of matches; here $P$ is the set of registered providers and $\mathcal{M}(r)$ is the set of matching providers). Then Requester has the option to choose which provider from set $P$ to contact for performing the service (the action send_request_to_provider with argument $p \in P$ representing the chosen provider). Finally, Requester waits for a reply from the contacted provider (the action receive_reply).

The Matchmaker agent registers and unregisters Provider offers and answers Requester requests for matching offers. Matchmaker informs Requester about available registered offers (the action tell). Note that $R e$ quester is responsible for choosing an appropriate matching offer from the available matching offers (the action send_request_to_provider). This complicates a bit the behavior of Requester compared with Front-agent and Broker cases (see Subsections 5.2 and 5.3).

Special care is taken to accurately model agent communication using FSP synchronization. The Matchmaker model requires alphabet extension (construct $\left\{\operatorname{tell}\left(r^{\prime} \in\right.\right.$ $\left.\left.R, P^{\prime} \subseteq \mathcal{P}\right)\right\}$ in Fig. 10 to correctly model communication between Matchmaker and Requester.

A critical situation may occur when a matching offer is found but the matching Provider chooses to cancel its offer by unregistering it with Matchmaker before it is actually contacted by Requester. This ability of Provider is modeled with the action refuse_request. Note that this situation cannot occur with Front-agent and Broker (remember that both Front-agent and Broker intermediate the request on behalf of Requester), so we did not have to model this ability of Provider in those cases.

5.2. Front-agent middle-agent in FSP. A Front-agent middle-agent (also known as Proxy, see Table 1) assumes that, before the interaction between the requester and the provider, requester preferences are known only to the requester, while provider capabilities are known both to provider and the middle-agent. This means that the provider will have to advertise its capabilities with Front-agent, and Front-agent is responsible for matching a request with registered capabilities advertisements. Additionally, as the provider capabilities will not be known to the requester, Front-agent also has the responsibility of intermediating the transaction between the requester and the matching provider (this is why often this type of middle-agent is called Broker rather than Front-agent; in our opinion a true Broker, is different, see below).

The block diagram and FSP specification of a system composed of requesters, providers and Front-agent are shown in Fig. 2.

The Provider agent is similar to the Matchmaker case. The Requester agent is simpler than the Matchmaker case: it submits a request to Front-agent (the action send_request) and then waits for Front-agent to either resolve that request (the action success) or fail (the action fail).

The Front-agent agent processes requests from Requester agents and registers offers from Provider agents. Note that, unlike Matchmaker, Front-agent has the responsibility of choosing an appropriate matching provider from the available matching providers $\mathcal{M}(r) \cap P$ (here $P$ is the set of registered providers and $\mathcal{M}(r)$ is the set of matching providers) using the action fragent_request and to resolve the request (the action fragent_request). Finally, the result is passed to the Requester agent using the action success. In conclusion, the actual Provider that fulfils the request for Requester on behalf of Front-agent is hidden from Requester that issued the request.

5.3. Broker middle-agent in FSP. A Broker middleagent (see Table 1) assumes that, before the interaction between the requester and the provider, requester preferences are known only to the requester and the middleagent and provider capabilities are known only to the provider and the middle-agent. The crucial point is, however, that requester preferences will not be known to the provider and provider capabilities will not be known to the requester. This means that Broker will truly intermediate transactions between providers and requesters in both directions: (i) if a requester submits a request either it cannot be matched and it is registered with Broker or it is matched with a provider capability and then transaction is intermediated by Broker, and (ii) if a provider advertises a capability then the capability is registered with Broker and it is also matched against registered requests; neither match is found and nothing more happens or matches are found and corresponding transactions are intermediated by Broker.

The block diagram and FSP specification of a system composed of requesters, providers and Broker are shown 


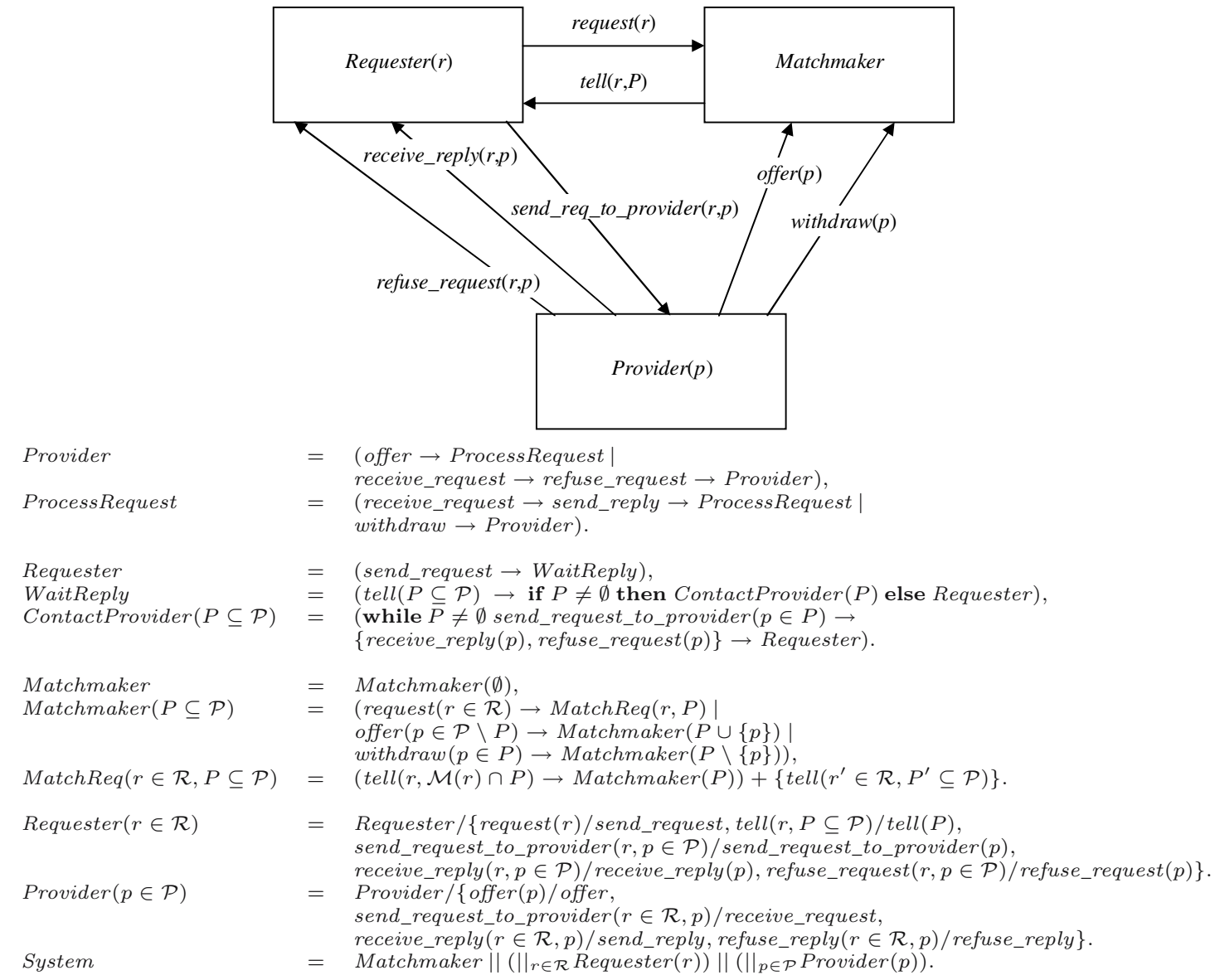

Fig. 1. System with the Matchmaker middle-agent.

in Fig. 3, Provider and Requester agents are similar to the Front-agent case.

Broker processes requests from Requesters and processes and registers offers from Providers. If a request can be served based on available matching offers, then Broker behaves similarly to Front-agent. Unlike Front-agent, if a request cannot be served based on currently registered offers, then, rather than reporting failure, the request is recorded until either (i) a new matching offer is registered with the Broker, or (ii) the request is deemed failed. Note that when a new offer is registered, Broker determines the set of recorded (i.e., not yet served) matching requests $-\mathcal{M}^{-1}(p) \cap R$ (note that $\mathcal{M}^{-1}(p)$ is the set of matching requesters and $R$ is the set of recorded requesters) and serves them using the matching provider $p-$ ContactProviderOff sub-process in Fig. 3.

5.4. System properties. A basic desirable property of systems with middle-agents is that they are free of deadlocks. The result is formally stated as follows.

Proposition 1. The systems with Matchmaker, Front- agent and Broker shown in Figs. 1 3 are deadlock free.

Proof. We consider only the proof for the Matchmaker system. The proofs for Front-agent and Broker follow the pattern and are therefore omitted.

Let us consider an arbitrary system state $S$. As the system is a parallel composition of processes, state $S$ is composed of sub-states corresponding to Matchmaker and to each of the Provider and Requester processes. Any progress from $S$ will be caused by an interaction between two processes: Matchmaker with Provider, Matchmaker with Requester or Requester with Provider. Note that Matchmaker can be in one of two states: (i) Matchmaker $(P)$ with $P \subseteq \mathcal{P}$; (ii) $\operatorname{MatchReq}(r, P)$ with $r \in \mathcal{R}$ and $P \subseteq \mathcal{P}$.

In the first case, this means that Matchmaker finalized to process a request and is waiting for a new one. If a new request is available, we are done. If Provider is available to register/unregister a capability offer, we are again done. Otherwise, this means that all requesters submitted requests to providers and wait for service. In this case, we randomly pick a pair Requester $(r)$ and $\operatorname{Provider}(p)$ with $r \in \mathcal{R}$ and $p \in \mathcal{P}$, and the system will proceed with interaction between Requester $(r)$ and $\operatorname{Provider}(p)$. 


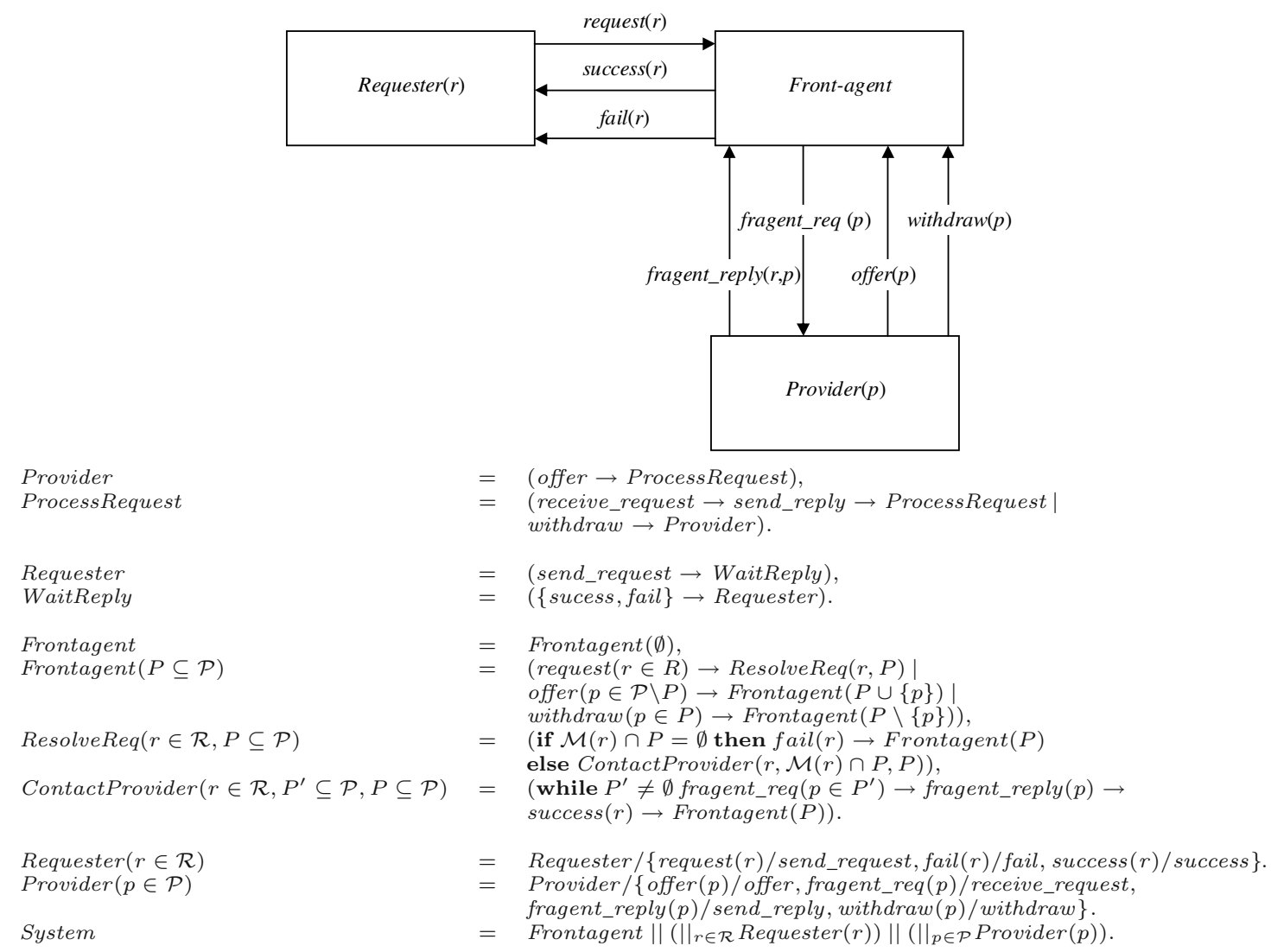

Fig. 2. System with the Front-agent middle-agent.

In the second case, progress will occur through interaction between Matchmaker and Requester $(r)$, as Requester $(r)$ is definitely in state WaitReply.

\section{FLTL modeling of system properties}

In this section we show how qualitative properties can be defined for systems with requesters, providers and middleagents. Clearly, there are many types of properties that can be defined for multi-agent systems, focusing either on typical features of agents like autonomy, proactiveness, reactiveness and adaptivity (Brazier et al., 2004), or on more specific aspects like those related to complex sequences of messages that agents may exchange during a negotiation process (Podorozhny et al., 2007). A full coverage of types of properties that are specific to each type of middleagent would require a more elaborated treatment and it is therefore beyond the scope of this paper. Nevertheless, in this section we exploit the FSP models introduced in the previous section by showing (using examples) how prototypical qualitative properties can be defined in temporal logic for systems with Matchmaker and Front-agent middle-agents (Bădică and Bădică, 2008a).

Formal modeling of agent systems has the advantage that models can be systematically checked against user- defined properties. A property is defined by a statement that should be true for all the possible execution paths of the system. A property is used to describe a desirable feature of the system behavior. The definition and analysis of properties of middle-agents have the advantage that they enable a formal and concise, rather than informal and speculative, comparison of types of middle-agents.

Properties of software systems are usually expressed as formulas in a temporal logic language (Clarke et al., 1986). Temporal logics are a subclass of modal logics specially tailored for declarative specification of properties of dynamic systems defined as labeled transition systems. Clearly, multi-agent systems that contain middleagents are a special class of software systems composed of many agents that dynamically interact and coordinate their actions by message exchange.

A property holds if the associated formula is true for all the possible executions of the system, as it is described by the system model. For system models captured using FSP it has been shown that a very convenient temporal logic for property expression is fluent linear temporal logic (FLTL) (Magee and Kramer, 2006).

In FLTL, primitive properties are expressed using $f l u$ ents. A fluent is a property whose truth is triggered by an 


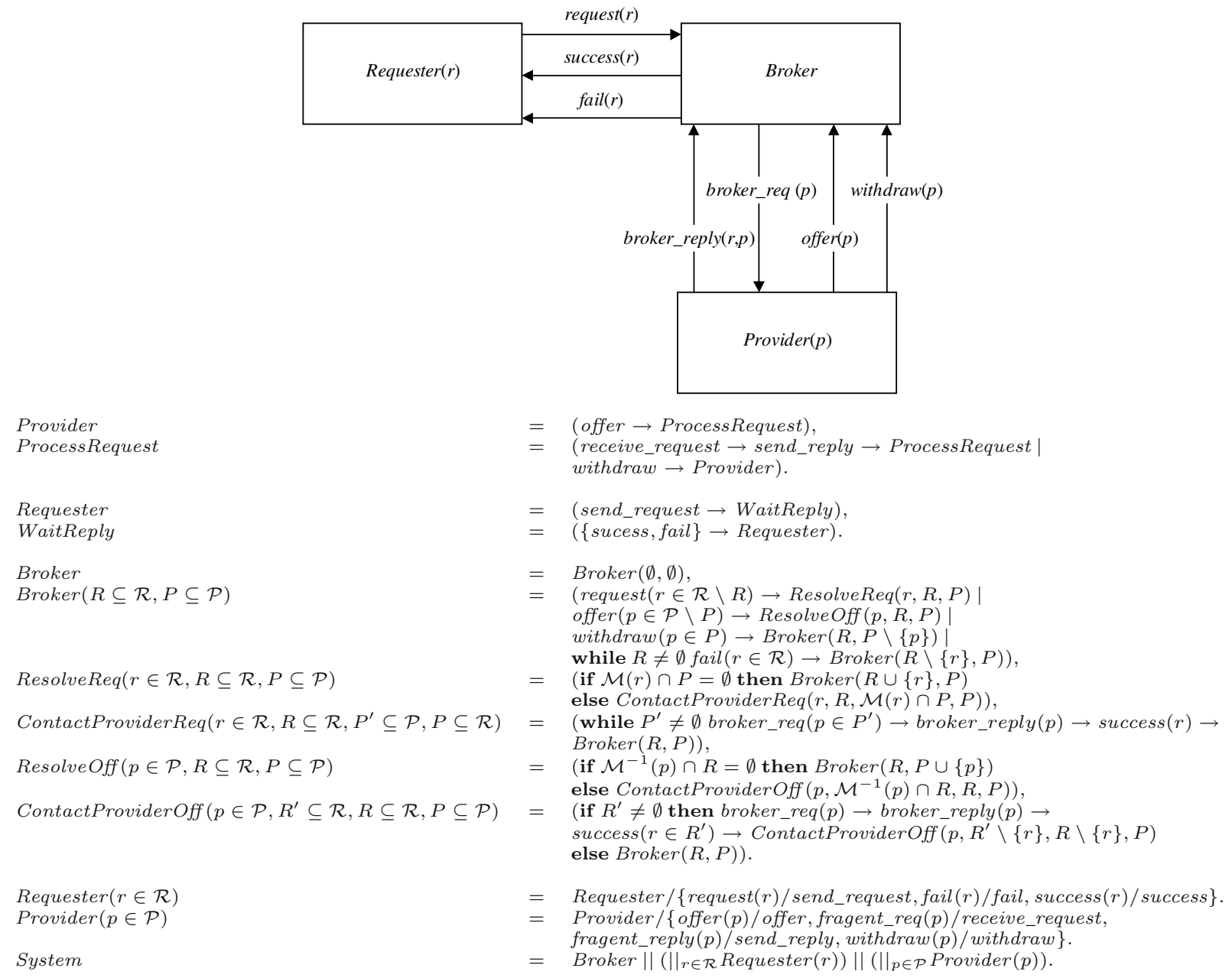

Fig. 3. System with the Broker middle-agent.

initiating event and that holds until the signalling of a terminating event. In FSP, it is natural to model initiating and terminating events by executing specific actions. Therefore, following (Magee and Kramer, 2006), a fluent is defined as a triple:

\section{fluent $F$}

$$
=\left\langle\left\{i_{1}, \ldots, i_{m}\right\},\left\{t_{1}, \ldots, t_{n}\right\}\right\rangle \text { initially } B,
$$

where (i) $\left\{i_{1}, \ldots, i_{m}\right\}$ and $\left\{t_{1}, \ldots, t_{n}\right\}$ are disjoint sets of initiating events and terminating events and (ii) $B$ is true or false and represents the initial value of $F$ (when the initial value $B$ of the fluent is not given, it is assumed to be false by default). When any of the initiating actions is observed, $F$ becomes true and remains so until any of the terminating actions is observed.

Every action $a$ defines a singleton fluent $F(a)$ having $a$ as the single initiating action and the rest of all actions as terminating actions as follows:

$$
\text { fluent } F(a)=\langle\{a\}, A \backslash\{a\}\rangle \text {. }
$$

A singleton fluent $F(a)$ is usually written as $a$ in FLTL formulas.

FLTL formulas are built over fluent propositions (including singleton fluents) using the usual logical operators $\wedge, \vee, \rightarrow, \neg$ and temporal operators $\mathbf{X}$ (next), $\mathbf{U}$ (until), $\mathbf{W}$ (weak until), $\mathbf{F}$ (eventually) and $\mathbf{G}$ (always) (Magee and Kramer, 2006). A property $P$ is specified using an FLTL formula $\Phi$ as follows:

$$
\text { assert } P=\Phi \text {. }
$$

Property specification was recognized as a difficult task requiring expert knowledge in formal methods, especially in temporal logic. However, according to the rigorous analysis performed by Dwyer et al. (1999), most of the specifications fall into the category of specification pat- 
terns. Therefore, in our work we have looked into the application of property specification patterns to the verification of Matchmaker and Front-agent middle-agents. In particular, we show how response properties that check if a given situation must always be followed by another situation can be applied to the verification of our system.

For the Matchmaker middle-agent, we can define a fluent that holds while Matchmaker is processing a request from a given Requester,

$$
\begin{aligned}
& \text { fluent } \begin{aligned}
M M_{-} P R O C \_R E Q(r \in \mathcal{R}) \\
\quad=\langle\{\text { request }(r)\},\{\operatorname{tell}(r, P \subseteq \mathcal{P})\}\rangle
\end{aligned}
\end{aligned}
$$

Similarly, we can define a fluent that holds while the system (Matchmaker and Provider) is processing a request from a given Requester. Note that this property captures also the intuition that the requester can finalize the processing immediately (without getting receive_reply or refuse_request) when there is no registered provider,

$$
\begin{aligned}
& \text { fluent } P R O C \_R E Q(r \in \mathcal{R}) \\
& =\langle\{\text { request }(r)\},\{\text { receive_reply }(r, p \in \mathcal{P}), \\
& \quad \text { refuse_request }(r, p \in \mathcal{P}), \operatorname{tell}(r, \emptyset)\}\rangle
\end{aligned}
$$

Similarly, we can define a fluent that holds while Front-agent is processing a request from a given Requester,

$$
\begin{aligned}
& \text { fluent } F A \_P R O C \_R E Q(r \in \mathcal{R}) \\
& =\langle\{\text { request }(r)\},\{\operatorname{success}(r), \operatorname{fail}(r)\}\rangle
\end{aligned}
$$

We can also describe the REGISTERED fluent that holds while Provider is registered with either Matchmaker or Front-agent (note that the REGISTERED fluent holds also for the Broker middle-agent),

$$
\begin{aligned}
& \text { fluent } R E G I S T E R E D(p \in \mathcal{P}) \\
& =\langle\{\text { offer }(r)\},\{\text { withdraw }(r)\}\rangle
\end{aligned}
$$

Using the REGISTERED $(p \in \mathcal{P})$ properties, we can define an indexed set of properties MATCHES $(r \in$ $\mathcal{R})$ that are true when there is a registered Provider that matches the given Requester $r \in \mathcal{R}$,

$$
\begin{aligned}
\text { assert MATCHES } & (r \in \mathcal{R}) \\
& =\vee_{p \in \mathcal{M}(r)} \operatorname{REGISTERED}(p)
\end{aligned}
$$

Using the defined fluents and formulas, we can express two characterizing response properties of a system with Front-agent: (i) "If a Requester issues a request to Front-agent, then Front-agent will eventually reply either with success or failure" and (ii) "If Requester issues a request to Front-agent and there is at least one matching Provider registered with Front-agent, then Front- agent will eventually reply with success". These properties can be formally defined using FLTL as follows:

$$
\begin{aligned}
& \text { assert FA_RESPONSE }(r \in \mathcal{R}) \\
& =\mathbf{G}(\text { request }(r) \rightarrow \\
& \left.\quad\left(F A \_P R O C \_R E Q(r) \mathbf{U}(\operatorname{success}(r) \vee \text { fail }(r))\right)\right) \\
& \text { assert FA_MATCHING_RESPONSE }(r \in \mathcal{R}) \\
& =\mathbf{G}((\text { request }(r) \wedge M A T C H E S(r)) \rightarrow \\
& \left.\quad\left(F A \_P R O C \_R E Q(r) \mathbf{U} \text { success }(r)\right)\right) .
\end{aligned}
$$

Different response properties can be defined for the Matchmaker middle-agent: "If Requester issues a request to Matchmaker, then Matchmaker will eventually reply with the set of matching providers",

$$
\begin{aligned}
& \text { assert } M M_{-} R E S P O N S E(r \in \mathcal{R}) \\
& =\mathbf{G}(\text { request }(r) \\
& \left.\rightarrow\left(M M_{-} P R O C_{-} R E Q(r) \mathbf{U} \vee_{P \subseteq \mathcal{M}(r)} \operatorname{tell}(r, P)\right)\right)
\end{aligned}
$$

We would be tempted to define a response property of the system with Matchmaker similar to Front-agent: "If there is a matching Provider offer already registered with Matchmaker when a request is issued by Requester, then the request will be served by a matching Provider",

$$
\begin{aligned}
& \text { assert MM_MATCHING_RESPONSE_BAD } \\
& (r \in \mathcal{R}) \\
& =\mathbf{G}((r e q u e s t(r) \wedge M A T C H E S(r)) \rightarrow \\
& \left.\left(P R O C \_R E Q(r) \mathbf{U} \text { receive_reply }(r, p \in \mathcal{P})\right)\right)
\end{aligned}
$$

Note, however, that this property does NOT hold! Between the time the matching offers are provided to Requester by Matchmaker and the time when Requester decides to contact a matching Provider, it may happen that the matching Provider decides to cancel the offer by unregistering with Matchmaker.

The correct reformulation of this property would require to guarantee that the system will either perform the request and reply accordingly (the action receive_reply) or will indicate explicitly that fulfilling the request was refused (the action refuse_request),

$$
\begin{aligned}
& \text { assert } M M_{-} \text {MATCHING_RESPONSE }(r \in \mathcal{R}) \\
& =\mathbf{G}((\text { request }(r) \wedge M A T C H E S(r)) \rightarrow \\
& \quad\left(P R O C \_R E Q(r) \mathbf{U}\{\text { receive_reply }(r, p \in \mathcal{P}),\right. \\
& \quad \text { refuse_request }(r, p \in \mathcal{P})\}))
\end{aligned}
$$

\section{Experiments and discussions}

We conducted a series of experiments to check the correctness of our models introduced in Section 5 As a side effect, we also recorded the size of the state model expressed as the number of states and transitions, depending on the number of requesters and providers. 
7.1. Experimental setup. Firstly we had to express the general models shown in Figs. 13 using the FSP/FLTL language supported by the LTSA tool (version 3.0) (Magee and Kramer, 2006). The main difficulty is the encoding of processes indexed with sets in the language supported by LTSA.

Assuming that $\mathcal{S}$ is a set with $n$ elements, the mapping of processes indexed with sets and/or set elements to the FSP notation supported by LTSA follows the guidelines: (i) an index $s \in \mathcal{S}$ is encoded as $[s]$; (ii) an index $S \subseteq$ $\mathcal{S}$ is encoded as $\left[s_{1}\right] \ldots\left[s_{n}\right]$ such that $s_{i}=1$ if $i \in S$ and $s_{i}=0$ if $i \notin S$. For example, ResolveReq $(2,\{1\},\{1,3\})$ is mapped to ResolveReq[2][1][0][1][0][1] and $\operatorname{tell}(1,\{2,3\})$ is mapped to tel1 [1] [0] [1] [1] .

Note that a mapping can be defined such that the size of the resulting FSP specification is linear in the product of number of providers with the number of requester 5 . This is an important desiderata to make the resulting FSP specification of a practical value.

Proposition 2. Let $m=|\mathcal{R}|$ and $n=|\mathcal{P}|$. The systems with Matchmaker, Front-agent and Broker shown in Figs. 13 can be mapped to the FSP language supported by the LTSA tool such that the size of the resulting specification is $O(m \times n)$.

Proof. The result is a consequence of the following two observations.

First note that the mapping of set indexed names of processes and actions produces new names of size linear with $m$ and $n$.

Second, the application of the following mapping rules produces parts of FSP specification that clearly have a size of $O(m \times n)$.

If $\operatorname{Proc}(S \subseteq \mathcal{S})$ is a set indexed process and $|\mathcal{S}|=n$, then the construct

$$
\operatorname{Proc}(S \subseteq \mathcal{S})=(\text { while } S \neq \emptyset \operatorname{action}(s \in S) \ldots)
$$

is mapped to (here assuming $n=3$ )

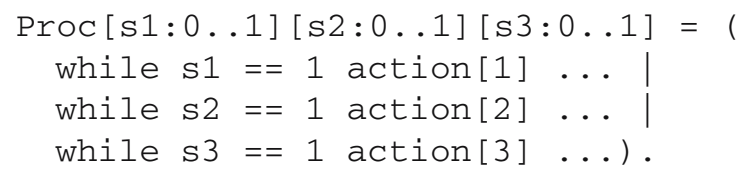

Note that the size of the resulting specification is $O(n)$.

If $\operatorname{Proc}_{i}(S \subseteq \mathcal{S}), i=1,2$, are set indexed processes and $|\mathcal{S}|=n$, then the construct

$$
\operatorname{Proc}_{1}(S \subseteq \mathcal{S})=\left(\text { while } S \neq \emptyset \operatorname{Proc}_{2}(S) \ldots\right)
$$

is mapped to (here assuming $n=3$ )

\footnotetext{
${ }^{5}$ Do not confuse the size of the FSP specification (i.e., the size of the FSP code measured, for example, as the number of nodes of its syntax tree) with the size of the LTS corresponding to this specification.
}

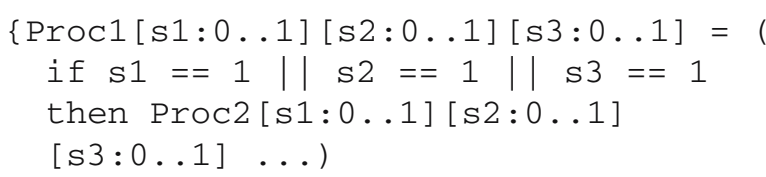

Note that the size of the resulting specification is $O(n)$.

If $\mathcal{S}_{1}$ and $\mathcal{S}_{2}$ are sets, $\left|\mathcal{S}_{1}\right|=m$ and $\left|\mathcal{S}_{2}\right|=n, \mathcal{M} \subseteq$ $\mathcal{S}_{1} \times \mathcal{S}_{2}$ is a relation, then the construct

$$
\begin{aligned}
& \operatorname{Proc}_{1}\left(s \in \mathcal{S}_{1}, S \subseteq \mathcal{S}_{2}\right)=(\text { if } \mathcal{M}(s) \cap S=\emptyset \text { then } \ldots \\
& \text { else } \left.\operatorname{Proc}_{2}(\mathcal{M}(s) \cap S) \ldots\right)
\end{aligned}
$$

is mapped to (here assuming $m=2, n=3$ and $\mathcal{M}=$ $\{(1,2),(1,3),(2,1),(2,2)\})$

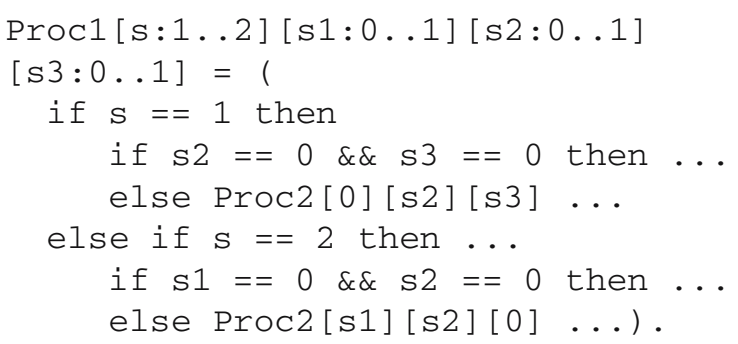

Note that the size of the resulting specification is $O(m \times n)$.

7.2. Results and discussion. In the experiments we considered three systems composed of: (i) $n$ requesters and $n+1$ providers, $2 \leq n \leq 5$; (ii) ones middle-agent per system (Matchmaker, Front-agent and respectively, Broker); and, (iii) the matching relation $\mathcal{M}=\{(i, i+1) \mid 1 \leq$ $i \leq n\} \cup\{(i, i+2) \mid 1 \leq i \leq n-1\} \cup\{n, 1)\}$.

We utilized model checking tools provided by LTSA to analyze the resulting FSP models of these systems that contain Matchmaker, Front-agent and Broker middleagents. The result of this analysis shows that these models are free of deadlocks, which is consistent with theoretical results (Section 5.4. Proposition 1). Sizes in terms of the number of states and transitions of their corresponding labeled transition systems are presented in Tables 24

For example, in Fig. 4 we present the encoding of the Front-agent system from Fig. 2] using the FSP notation supported by the LTSA tool. Note that this system contains one Front-agent, two Requester agents and three Provider agents. The matching function is defined as follows: $\mathcal{M}=\{(1,2),(1,3),(2,3),(2,1)\}$, i.e., Requesterl will match with Provider 2 and Provider3, and Requester 2 will match with Provider3 and Providerl. Referring to Fig. 4 , the fact that the request of Requesterl must match with Provider 2 and Provider 3 is modeled as follows:

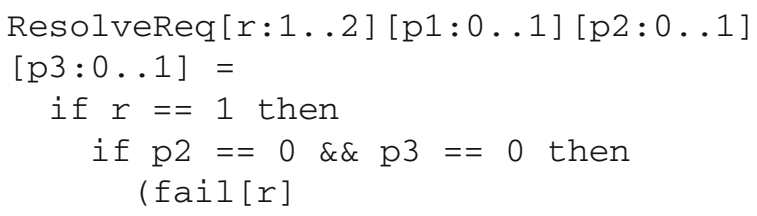


Table 2. LTS size of the system with the Matchmaker middle-agent.

\begin{tabular}{|c|c|c|c|c|}
\hline \# requesters & \# providers & \# states & \# transitions & \# states after minimization \\
\hline \hline 2 & 3 & 608 & 2272 & 376 \\
\hline 3 & 4 & 3392 & 14720 & 2064 \\
\hline 4 & 5 & 166400 & 1010176 & N/A \\
\hline 5 & 6 & $>1000000$ & $>7000000$ & N/A \\
\hline
\end{tabular}

Table 3. LTS size of the system with the Front-agent middle-agent.

\begin{tabular}{|c|c|c|c|c|}
\hline \# requesters & \# providers & \# states & \# transitions & \# states after minimization \\
\hline \hline 2 & 3 & 56 & 92 & 52 \\
\hline 3 & 4 & 160 & 268 & 148 \\
\hline 4 & 5 & 416 & 704 & 384 \\
\hline 5 & 6 & 1024 & 1744 & 944 \\
\hline
\end{tabular}

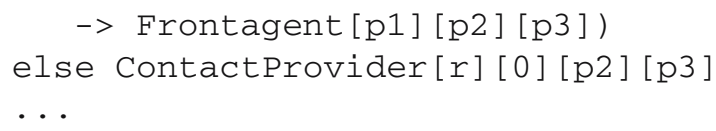

This means that when $r=1$, i.e., Front-agent received a request from Requester1 and neither Provider 2 nor Provider 3 are registered (i.e., $p 2=0$ and $p 3=0$ ), Front-agent will return failure, otherwise it will proceed to serving the request.

7.3. Experiments with properties verification. We also conducted model checking experiments with LTSA to check our models of Matchmaker and Frontagent against the FLTL qualitative properties introduced in Section 6. We updated our mapping framework with the mapping of FLTL formulas. For example, the response properties $M M_{-} R E S P O N S E$, $M M \_M A T C H I N G \_R E S P O N S E \_B A D$, as well as $M M \_M A T C H I N G \_R E S P O N S E$ for Matchmaker are shown in Fig. 5] (assuming there are four requesters, five providers and $\mathcal{M}(2)=\{3,4\}$ )

We utilized the LTSA tool to check the property $M M \_M A T C H I N G \_R E S P O N S E \_B A D$ for $r=2$. We obtained the trace presented in Fig. 6 that shows that the property does not hold.

More precisely, taking into account that in this system there are four requesters and five providers, this trace points out to the following system execution scenario: Providers 1, 2 and 3 register their offers with Matchmaker; Requester 2 queries Matchmaker, which consequently responds with a single matching offer $-\{3\}$ (remember that in this example Requester 2 matches with Providers 3 and 4); Provider 3 then cancels the offer; finally, Requester 2 contacts Provider 3, which however, has to refuse the request, as it was canceled before. Summarizing, when request [2] occurred, MATCHES [2 ]

${ }^{6}$ FSP and FLTL models used in experiments are available at http://software.ucv.ro/ cbadica/fsp/amcs10_models.zip. was true (as REGISTERED [3 ] was true), while the fluent PROC_REQ[2] ceased to be true before the occurrence of an event receive_reply[2] [p] with $1 \leq p \leq 5$, as it was required by the property MM_RESPONSE2_BAD.

Our conclusion after applying LTSA to checking FSP models of middle-agents is that, despite the usefulness of model checking to verify simple models of middle agents, the technique is limited for at least two reasons: (i) the approach is not general because it is only able to check specific instances of systems with a given number of agents and a specific matching relation rather than general models; (ii) the state-space explosion problem hinders the analysis of systems with a larger number of states.

Nevertheless, we think that the developed models are useful to better understand available types of middleagents and to enable a theoretical study of their qualitative properties, which would be obviously more robust than the experimental investigation using model checking.

\section{Conclusions}

Our work sheds light on the modeling of software systems incorporating middle-agents using process algebra for defining the semantics of the different actors in such applications. In particular, we proposed a formal framework based on FSP process algebra and FLTL temporal logic for the modeling and verification of systems that contain requesters, providers and middle-agents. We applied this framework to model and verify systems with three types of middle-agents: Matchmaker, Front-agent, and Broker. For each system, we defined a sample set of qualitative properties expressed in FLTL and we checked the resulting models against these properties with the help of the LTSA analysis tool. While this work might look incomplete with respect (i) to the coverage of the set of nine types of middle-agents that were identified in the literature, and (ii) to considering only those properties that are formally expressible using FLTL, it still has a sen- 
Table 4. LTS size of the system with the Broker middle-agent.

\begin{tabular}{|c|c|c|c|c|}
\hline \# requesters & \# providers & \# states & \# transitions & \# states after minimization \\
\hline \hline 2 & 3 & 293 & 464 & 281 \\
\hline 3 & 4 & 1788 & 2997 & 1718 \\
\hline
\end{tabular}

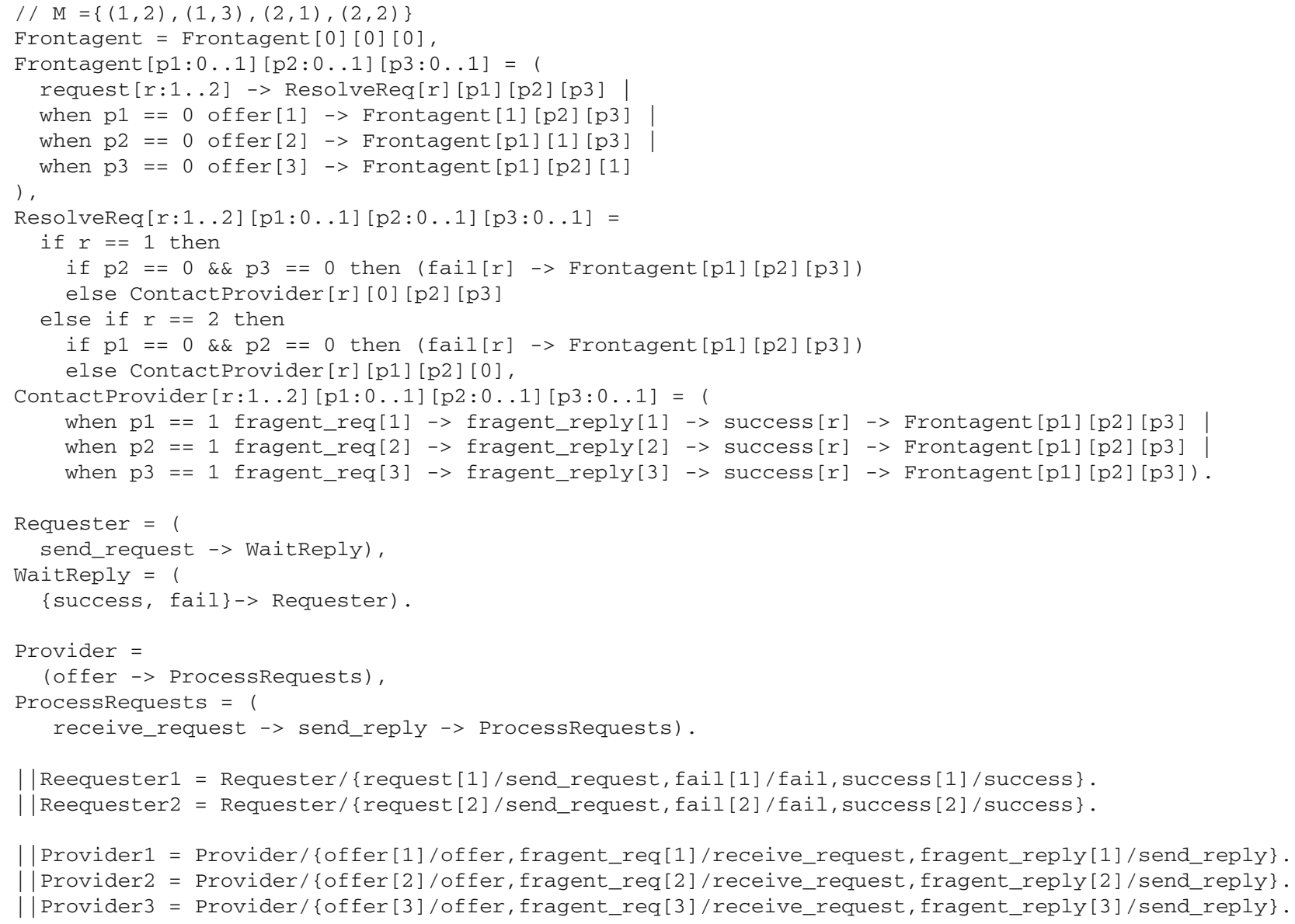

| System = (Frontagent || Reequester1 || Reequester2 || Provider1 || Provider2 || Provider3).

Fig. 4. Encoding of the Front-agent model with $n=2$ for the LTSA tool.

const False $=0$

const $\mathrm{R}=4$

const $\mathrm{P}=5$

fluent REGISTERED $[\mathrm{p}: 1 \ldots \mathrm{P}]=$

$<\{$ offer $[p]\},\{$ withdraw $[p]\}>$ initially False

fluent PROC_REQ $[r: 1 \ldots R]=$

$<\{$ request $[r]\},\left\{r e c e i v e \_r e p l y[r][p: 1 \ldots P]\right.$, refuse_request $\left.[r][p: 1 \ldots P]\right\}>$ initially False

fluent MATCHMAKER_PROC_REQ $[r: 1 \ldots R]=$

$<\{r e q u e s t[r]\},\{t e l 1[r][p 1: 0 \ldots 1][p 2: 0 \ldots 1][p 3: 0 \ldots 1][p 4: 0 \ldots 1][p 5: 0 \ldots 1]\}>$ initially False

assert MATCHES2 =

(REGISTERED[3] || REGISTERED[4])

assert MM_RESPONSE2 =

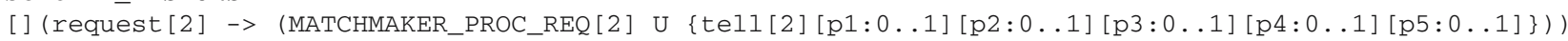
assert MM_MATCHING_RESPONSE2_BAD =

[] (request [2] \&\& MATCHES2 $\rightarrow$ (PROC_REQ[2] U receive_reply[2] [p:1..P]))

assert MM_MATCHING_RESPONSE2 $=$

[] (request[2] \&\& MATCHES2 -> (PROC_REQ[2] U \{receive_reply[2][p:1..P], refuse_request[2][p:1..P]\}))

Fig. 5. Encodings of system properties for the LTSA tool. 


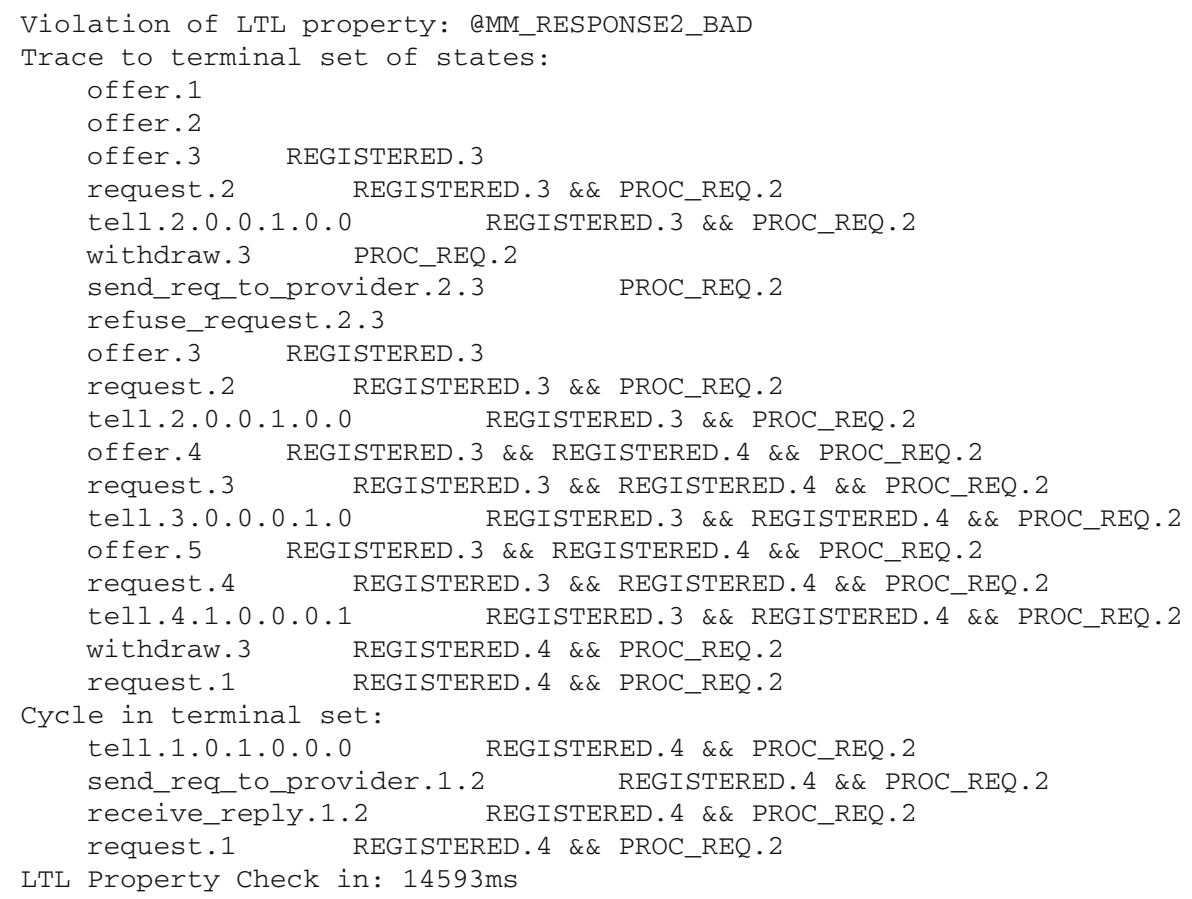

Fig. 6. Trace of property verification using LTSA.

se of completeness by a full treatment of the modelingspecification-verification cycle of a software system. In the future, we intend to (i) model more complex systems containing all types of middle-agents using a compositional approach; (ii) extend the framework for properties specification and verification with a comprehensive analysis of properties specific to all nine types of middle-agents.

\section{Acknowledgment}

The work reported in this paper was partly supported by (i) the research project SCIPA: Servicii software semantice de Colaborare si Interoperabilitate pentru realizarea Proceselor Adaptive de business between the Software Engineering Department, University of Craiova, Romania, and the National Authority for Scientific Research, Romania, and partly by (ii) the research project Agent-Based Service Negotiation in Computational Grids between the Systems Research Institute, Polish Academy of Sciences, and the Software Engineering Department, University of Craiova, Romania.

We would like to thank the guest editors, Prof. Antoni Ligęza and Dr. Grzegorz J. Nalepa, for the effort made to prepare this special section and also the anonymous reviewers for their very useful feedback to improve the preliminary version of this paper.

\section{References}

Alagar, V. and Holliday, J. (2002). Agent types and their formal descriptions, Technical Report COEN-2002-09-19A, Santa Clara University, Santa Clara, CA.
Bergstra, J., Ponse, A. and Smolka, S. (2001). Handbook of Process Algebra, Elsevier Science, Amsterdam.

Brazier, F.M.T., Cornelissen, F., Gustavsson, R., Jonker, C.M., Lindeberg, O., Polak, B. and Treur, J. (2004). Compositional verification of a multi-agent system for one-to-many negotiation, Applied Intelligence 20(2): 95-117.

Bădică, A. and Bădică, C. (2008a). Conceptualizing interactions with matchmakers and front-agents using formal verification methods, in D. Dochev, M. Pistore and P. Traverso (Eds.), Proceedings of the 13th International Conference on Artificial Intelligence: Methodology, Systems, Applications (AIMSA 2008), Lecture Notes in Artificial Intelligence, Vol. 5253, Springer-Verlag, Berlin, pp. 390-394.

Bădică, A. and Bădică, C. (2008b). Formal specification of matchmakers, front-agents, and brokers in agent environments using FSP, Proceedings of the 6th International Workshop on Modelling, Simulation, Verification and Validation of Enterprise Information Systems (MSVVEIS 2008), Barcelona, Spain, pp. 9-18.

Bădică, A. and Bădică, C. (2008c). Formalizing agent-based english auctions using finite state process algebra, Journal of Universal Computer Science 14(7): 1118-1135.

Bădică, A. and Bădică, C. (2008d). Modeling interactions in agent-based English auctions with matchmaking capabilities, in C. Bădică, G. Mangioni, V. Carchiolo and D.D. Burdescu (Eds.), Intelligent Distributed Computing, Systems and Applications (IDC 2008), Studies in Computational Intelligence, Vol. 162, Springer-Verlag, Berlin, pp. 45-54.

Bădică, A. and Bădică, C. (2008e). Specification and verification of agent interactions in matchmaking processes using FSP 
and FLTL, Proceedings of the 23rd International Symposium on Computer and Information Sciences (ISCIS 2008), Istanbul, Turkey, pp. 1-6.

Bădică, A. and Bădică, C. (2009a). Formalizing agent interactions in matchmaking processes using FSP and FLTL, Scientific Bulletin of "Politehnica" University of Timisoara, Transactions on Automatic Control and Computer Science 54(68)(1): 13-18.

Bădică, A. and Bădică, C. (2009b). Specification and verification of an agent-based auction service, in G.A. Papadopoulos, W. Wojtkowski, G. Wojtkowski, S. Wrycza, and J. Zupancic (Eds.) Information System Development. Towards a Service Provision Society (ISD 2008), Springer-Verlag, New York, NY, pp. 239-248.

Bădică, A., Bădică, C. and Liţoiu, L. (2003). Role activity diagrams as finite state processes, Proceedings of the 2 nd International Symposium on Parallel and Distributed Computing (ISPDC 2003), Ljubljana, Slovenia, pp. 15-22.

Bădică, A., Bădică, C. and Liţoiu, L. (2007). Middle-agents interactions as finite state processes: Overview and example, Proceedings of the 16th IEEE International Workshop on Enabling Technologies: Infrastructure for Collaborative Enterprises (WETICE 2007), Paris, France, pp. 12-17.

Bădică, A., Bădică, C., Popescu, E. and Scafes, M. (2009). A process algebraic framework for service coordination, Proceedings of the 5th International Symposium on Applied Computational Intelligence and Informatics (SACI 2009), Timișoara, Romania, pp. 515-520.

Bădică, C., Ganzha, M. and Paprzycki, M. (2007). Developing a model agent-based e-commerce system, in $\mathrm{J}$. Lu, G. Zhang and D. Ruan (Eds.), E-Service Intelligence: Methodologies, Technologies and Applications, Studies in Computational Intelligence, Vol. 55, Springer-Verlag, Berlin, pp. 555-578.

Clarke, E.M., Emerson, E.A. and Sistla, A.P. (1986). Automatic verification of finite-state concurrent systems using temporal logic specifications, ACM Transactions on Programming Languages and Systems 8(2): 244-263.

Decker, K., Sycara, K.P. and Williamson, M. (1997). Middleagents for the internet, Proceedings of the 15th International Joint Conference on Artificial Intelligence (IJCAI'97), Nagoya, Aichi, Japan, Vol. 1, pp. 578-583.

D'Ippolito, N., Fischbein, D., Chechik, M. and Uchitel, S. (2008). MTSA: The modal transition system analyser, Proceedings of the 23rd IEEE/ACM International Conference on Automated Software Engineering, ASE'08, L'Aquila, Italy, pp. 475-476.

Dobriceanu, A., Bîscu, L., Bădică, A. and Bădică, C. (2009). The design and implementation of an agent-based auction service, International Journal of Agent Oriented Software Engineering 3(2/3): 188-211.

Dwyer, M.B., Avrunin, G.S. and Corbett, J.C. (1999). Patterns in property specifications for finite-state verification, Proceedings of the 21st International Conference on Software Engineering (ICSE 1999), Los Angeles, CA, USA, pp. 411-420.
Esterline, A., Rorie, T. and Homaifar, A. (2006). A processalgebraic agent abstraction, in C.A. Rouff, M. Hinchey, J. Rash, W. Truszkowski and D. Gordon-Spears (Eds.) Agent Technology from a Formal Perspective, NASA Monographs in Systems and Software Engineering, SpringerVerlag, London, pp. 88-137.

Fasli, M. (2007). Agent Technology for E-Commerce, John Wiley $\&$ Sons, Chichester

Fokkink, W. (2007). Texts in Theoretical Computer Science, EATCS Series, Springer-Verlag, Berlin/Heidelberg.

Foster, H., Uchitel, S., Magee, J. and Kramer, J. (2006). LTSAWS: A tool for model-based verification of web service compositions and choreography, in L.J. Osterweil, H.D Rombach and M.L. Soffa (Eds.), Proceedings of the 28th International Conference on Software Engineering (ICSE 2006), Shanghai, China, pp. 771-774.

Goh, S., Chhetri, M.B. and Kowalczyk, R. (2007). JADE-FSMengine: A deployment tool for flexible agent behaviours in JADE, Proceedings of the 2007 IEEE/WIC/ACM International Conference on Intelligent Agent Technology, IAT'2007, Silicon Valley, CA, USA, pp. 524-527.

Hennicker, R. and Ludwig, M. (2005). Property-driven development of a coordination model for distributed simulations, in M. Steffen and G. Zavattaro (Eds.), Formal Methods for Open Object-Based Distributed Systems, Lecture Notes in Computer Science, Vol. 3535, Springer, Berlin/Heidelberg, pp. 290-305.

Hoare, C.A.R. (1985). Communicating Sequential Processes Prentice Hall International Series in Computer Science, Upper Saddle River, NJ.

Hristozova, M. and Sterling, L. (2003). Experiences with ontology development for value-added publishing, in S. Cranefield, T.W. Finin, V.A.M. Tamma and S. Willmott (Eds.), Proceedings of the International Workshop on Ontologies in Agent Systems (OAS 2003), CEUR Workshop Proceedings, Vol. 73, pp. 17-24.

Klusch, M. and Sycara, K.P. (2001). Brokering and matchmaking for coordination of agent societies: A survey, in A. Omicini, F. Zambonelli, M. Klusch and R. Tolksdorf (Eds.), Coordination of Internet Agents: Models, Technologies, and Applications, Springer, Berlin/Heidelberg/New York, NY, pp. 197-224.

Magee, J. and Kramer, J. (2006). Concurrency. State Models and Java Programs, 2nd Edn., John Wiley \& Sons, Chichester.

Mbala, A., Padgham, L. and Winikoff, M. (2006). Design options for subscription managers, in G.A. Vouros and T. Panayiotopoulos (Eds.), Agent-Oriented Information Systems III, Lecture Notes in Computer Science, Vol. 3529, Springer, London, pp. 259-274.

Merayo, M., Núñez, M., and Rodríguez, I. (2007). Specification of multi-agent systems by using EUSMs, Proceedings of the International Symposium on Fundamentals of Software Engineering (FSEN'2007), Lecture Notes in Computer Science, Vol. 4767, Springer, Berlin/Heidelberg/New York NY, pp. 318-333. 
Miller, T. and McBurney, P. (2007). Using constraints and process algebra for specification of first-class agent interaction protocols, Engineering Societies in the Agents World VII (ESAW 2006), Lecture Notes in Computer Science, Vol. 4457, Springer, Berlin/Heidelberg/New York, NY, pp. 245-254.

Milner, R. (1999). Communicating and Mobile Systems: The $\pi$-Calculus, Cambridge University Press, Cambridge.

Podorozhny, R.M., Khurshid, S., Perry, D.E. and Zhang, X. (2007). Verification of multi-agent negotiations using the alloy analyzer, Proceedings of the 6th International Conference Integrated Formal Methods (IFM'2007), Lecture Notes in Computer Science, Vol. 4591, Springer, Berlin/Heidelberg/New York, NY, pp. 501-517.

Rahimi, S., Cobb, M., Ali, D. and Petry, F. (2002). A modeling tool for intelligent-agent based systems: Api-calculus, in V. Loia (Ed.), Soft Computing Agents: A New Perspective for Dynamic Systems, Frontiers in Artificial Intelligence and Applications, IOS Press, Amsterdam, pp. 165-186.

Rouff, C., Rash, J., Hinchey, M. and Truszkowski, W. (2006). Formal methods at NASA Goddard Space Flight Center, in C.A. Rouff, M. Hinchey, J. Rash, W. Truszkowski and D. Gordon-Spears (Eds.), Agent Technology from a Formal Perspective, NASA Monographs in Systems and Software Engineering, Springer-Verlag, London, pp. 287-309.

Wang, F. (2002). Self-organising communities formed by middle agents, Proceedings of the 1st International Joint Conference on Autonomous Agents and Multiagent Systems, AAMAS'02, Bologna, Italy, pp. 1333-1339.

Wong, H.C. and Sycara, K. (2000). A taxonomy of middleagents for the Internet, Proceedings of the 4th International Conference on MultiAgent Systems (ICMAS-2000), Boston, MA, USA, pp. 465-466.

Xu, D.-X., El-Ariss, O., Xu, W.-F. and Wang, L.-Z. (2009). Aspect-oriented modeling and verification with finite state machines, Journal of Computer Science and Technology 24(5): 949-961.

Yarom, I., Rosenschein, J.S. and Goldman, C.V. (2003). The role of middle-agents in electronic commerce, IEEE Intelligent Systems 18(6): 15-21.
Zhang, P., Muccini, H. and Li, B. (2010). A classification and comparison of model checking software architecture techniques, Journal of Systems and Software 83(5): 723-744.

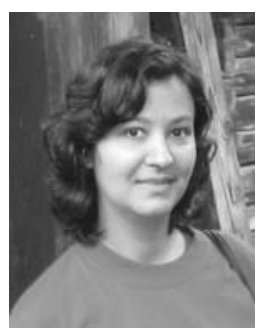

Amelia Bădică holds a Ph.D. in economics and she currently works as a senior lecturer at the $\mathrm{Bu}$ siness Information Systems Department, University of Craiova, Romania. She has specialization in management information systems obtained at Binghamton University, USA. Her research interests cover the application of expert systems, software engineering and Web technologies in business and management. She has co-authored several papers in journals and conference proceedings on these subjects. She has been involved as the principal investigator in a research project concerning data extraction from the Web.

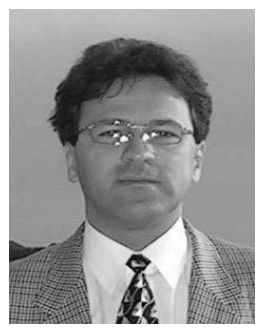

Costin Bădică holds a Ph.D. in computer science, and in 2006 he received the title of a professor of computer science from the University of Craiova. He is currently with the Department of Software Engineering, Faculty of Automatics, Computers and Electronics of the University of Craiova, Romania. In 2001 and 2002 he was a post-doctoral fellow with the Department of Computer Science, King's College London, UK. His research interests are at the intersection of artificial intelligence, distributed systems and software engineering. He has authored and co-authored more than 100 publications related to these topics in the form of journal articles, book chapters and conference papers. He has prepared special journal issues and co-edited four books in Springer's Studies in Computational Intelligence series. $\mathrm{He}$ co-initiated and is co-organizing the Intelligent Distributed Computing $(I D C)$ series of international conferences held annually. He is a member of the editorial boards of four international journals. He has also served as programme committee member of numerous international conferences.

Received: 12 April 2010

Revised: 21 November 2010 